PROCEEDINGS OF THE

AMERICAN MATHEMATICAL SOCIETY

Volume 139, Number 12, December 2011, Pages 4235-4245

S 0002-9939(2011)10858-X

Article electronically published on April 27, 2011

\title{
A FORMULA FOR THE $*$-CORE OF AN IDEAL
}

\author{
LOUIZA FOULI, JANET C. VASSILEV, AND ADELA N. VRACIU
}

(Communicated by Bernd Ulrich)

\begin{abstract}
Expanding on the 2010 work of Fouli and Vassilev, we determine a formula for the $*$-core of an ideal in two different settings: (1) in a CohenMacaulay local ring of characteristic $p>0$, with perfect residue field and test ideal of depth at least two, where the ideal has a minimal $*$-reduction that is a parameter ideal, and (2) in a normal local domain of characteristic $p>0$, with perfect residue field and $\mathfrak{m}$-primary test ideal, where the ideal is a sufficiently high Frobenius power of an ideal. We also exhibit some examples where our formula fails if our hypotheses are not met.
\end{abstract}

\section{INTRODUCTION}

In a recent paper [FV], Fouli and Vassilev introduced the $c l$-core of an ideal for a closure operation $c l$. Like the core of an ideal originally defined by Rees and Sally [RS], the $c l$-core of an ideal is the intersection of all the $c l$-reductions of the ideal, where $J$ is a $c l$-reduction of an ideal $I$ if $J \subseteq I$ and $J^{c l}=I^{c l}$. Of particular interest for rings of characteristic $p>0$ is the $*$-core, where $*$ denotes tight closure.

The tight closure of an ideal is contained in the integral closure but is generally much closer to the ideal than the integral closure. Note that a $*$-reduction of an ideal $I$ is also a reduction. Hence, $\operatorname{core}(I) \subseteq *$-core $(I)$. Recall that core $(I)$ sits deep within $I ; *$-core $(I)$ will lie within $I$ but will be generally much closer to $I$. In [FV], Fouli and Vassilev determined that the $*$-core and the core of an ideal $I$ agree if the analytic spread of $I$ is equal to the $*$-spread of $I$, where the $*$-spread is the minimal number of generators of any minimal $*$-reduction of $I$. Fouli and Vassilev also exhibited some examples where the core and the $*$-core of an ideal are not equal.

Huneke and Swanson [HS1 determined that in a 2-dimensional regular local ring the core of an integrally closed ideal $I$ is given by $\operatorname{core}(I)=(J: I) I$, where $J$ is a minimal reduction of $I$. This result was later generalized by Corso, Polini and Ulrich CPU2 under suitable assumptions on $I$ (not necessarily integrally closed) in the case when $(R, \mathfrak{m})$ is a Gorenstein local ring. In particular, if $I$ is an $\mathfrak{m}-$ primary ideal with reduction number at most 1, then this formula holds CPU2, Theorem 2.6]. We will discuss the additional assumptions on $I$ in Section 2,

Various authors have developed techniques for studying the core of an ideal (see for example [CPU1, [CPU2], [HT], [PU], HySm1, HySm2]). However, these

Received by the editors October 23, 2009 and, in revised form, October 22, 2010.

2010 Mathematics Subject Classification. Primary 13A30, 13A35, 13B22.

Key words and phrases. Tight closure, reduction, core, *-independent, spread.

The second author was partly supported by the NSA grant H98230-09-1-0057. 
techniques do not apply when studying the $*$-core and minimal $*$-reductions. For one, the analytic spread of an ideal $\ell(I)$ is always bounded above by the dimension of the ring and the $*$-spread can grow arbitrarily large. In order to better understand minimal $*$-reductions and the $*$-core of an ideal we make use of special tight closure techniques introduced by Vraciu [Vr1] and further developed in [ $\mathrm{HV}$, [Ep, and EV]. To work with special tight closure, we need to assume that $R$ is an excellent normal local ring of characteristic $p>0$ with perfect residue field. Using techniques of special tight closure we obtain a formula for the $*$-core of an ideal that is very similar to the formula shown by Corso, Polini and Ulrich in [CPU2, Theorem 2.6]; however, with minimal $*$-reductions instead of minimal reductions. The ideals we consider in this paper have reduction number equal to one with respect to their minimal $*$-reductions.

The outline of the paper is as follows: In Section 2, we briefly introduce the reader to tight closure and conditions associated to the study of the core. In Section 3, over an excellent normal local ring, we provide two criteria that when satisfied establish that $*$-core $(I)=(J: I) J=(J: I) I$, where $J$ is any minimal $*$-reduction of an ideal $I$ (Theorem 3.4). We subsequently use these criteria to show that when $R$ is a Cohen-Macaulay, normal, excellent local ring of characteristic $p>0$ with perfect residue field and test ideal of depth at least two, then $*$-core $(I)=$ $(J: I) J=(J: I) I$, where $I$ is an ideal that has a minimal $*$-reduction $J$ generated by part of a system of parameters (Theorem 3.7). If $(R, \mathfrak{m})$ is a normal local ring of characteristic $p>0$ and test ideal equal to $\mathfrak{m}$, we show that $*$-core $(I)=$ $(J: I) J=(J: I) I$, where $I$ is an ideal and $J$ is any minimal $*$-reduction of $I$ (Theorem 3.10). Furthermore, when $(R, \mathfrak{m})$ is a normal local ring of characteristic $p>0$, with perfect residue field and $\mathfrak{m}$-primary test ideal, we determine that *-core $\left(I^{[q]}\right)=\left(I^{[q]}: J^{[q]}\right) J^{[q]}$ for $q$ a sufficiently large power of $p$ and for every minimal $*$-reduction $J$ of $I$ (Theorem 3.12). In Section 4, we exhibit two examples where our formula fails, suggesting that if the criteria of Theorem 3.4 are not satisfied, then the conclusion need not hold.

\section{Preliminaries}

Since the $*$-core of an ideal $I$ is based on reductions stemming from the tight closure of $I$, we will review some tight closure concepts to help clarify these notions for the reader. For a more extensive introduction, see [Hu] or [HH1].

Definition 2.1. Let $R$ be a Noetherian ring of characteristic $p>0$. We denote positive powers of $p$ by $q$ and the set of elements of $R$ which are not contained in the union of minimal primes by $R^{0}$. Then

(a) For any ideal $I \subseteq R, I^{[q]}$ is the ideal generated by the $q$ th powers of elements in $I$.

(b) We say an element $x \in R$ is in the tight closure, $I^{*}$, of $I$ if there exists a $c \in R^{0}$ such that $c x^{q} \in I^{[q]}$ for all large $q$.

Finding the tight closure of an ideal would be hard without test elements and test ideals. A test element is an element $c \in R^{0}$ such that $c I^{*} \subseteq I$ for all $I \subseteq R$. Note that $c \in \bigcap_{I \subseteq R}\left(I: I^{*}\right)$. The ideal $\tau=\bigcap_{I \subseteq R}\left(I: I^{*}\right)$ is called the test ideal of $R$.

In a Noetherian local ring of characteristic $p>0$, Vraciu Vr1] defined the special tight closure, $I^{* s p}$, to be the elements $x \in R$ such that $x^{q_{0}} \in\left(\mathfrak{m} I^{\left[q_{0}\right]}\right)^{*}$ for some 
$q_{0}=p^{e_{0}}$. Vraciu shows that $I^{* s p} \cap I=\mathfrak{m} I$ if $I$ is generated by $*$-independent elements Vr1, Proposition 4.2]. Furthermore, Huneke and Vraciu show in [HV, Theorem 2.1] that $I^{*}=I+I^{* s p}$, without the assumption that $I$ is generated by $*$-independent elements. Recall that the elements $f_{1}, \ldots, f_{n}$ are said to be *-independent if $f_{i} \notin\left(f_{1}, \ldots, \hat{f}_{i}, \ldots, f_{n}\right)^{*}$ for all $i=1, \ldots, n$.

Note that the minimal $*$-reductions of $I$ are generated by $*$-independent elements. In particular, Epstein shows that if $R$ is an excellent analytically irreducible local domain, then every minimal $*$-reduction is generated by the same minimal number of generators, namely the $*$-spread of $I[\mathrm{Ep}$, Theorem 5.1]. He also showed that $I^{* s p}=J^{* s p}$ for all $*$-reductions $J$ of $I$ [Ep, Lemma 3.4].

We mention here the following result of Aberbach that is used in many arguments in this paper.

Proposition 2.2 ([Ab, Proposition 2.4]). Let $(R, \mathfrak{m})$ be an excellent, analytically irreducible local ring of characteristic $p>0$, let $I$ be an ideal, and let $f \in R$. Assume that $f \notin I^{*}$. Then there exists $q_{0}=p^{e_{0}}$ such that for all $q \geq q_{0}$ we have $I^{[q]}: f^{q} \subseteq \mathfrak{m}^{\left[q / q_{0}\right]}$.

We conclude the section by recalling some definitions and conditions associated with the study of the core. We also provide some instances for when these conditions are satisfied.

Let $(R, \mathfrak{m})$ be a Noetherian local ring and let $I$ be an ideal in $R$. Let $d=\operatorname{dim} R$.

(a) The analytic spread $\ell=\ell(I)$ is the dimension of the special fiber ring of $I$, $\mathcal{F}(I)=R[I t] / \mathfrak{m} R[I t]=R / \mathfrak{m} \oplus I / \mathfrak{m} I \oplus I^{2} / \mathfrak{m} I^{2} \oplus \ldots$. It is well known that $\ell(I)=\mu(J)$ for every minimal reduction $J$ of $I$, where $\mu(J)$ denotes the minimal number of generators of $J[\mathrm{NR}$.

(b) $I$ satisfies $G_{s}$ if $\mu\left(I_{\mathfrak{p}}\right) \leq s-1$ for every prime $\mathfrak{p} \supseteq I$, with $\operatorname{dim} R_{\mathfrak{p}} \leq s-1$. If $I$ is $\mathfrak{m}$-primary, then $I$ satisfies $G_{d}$ automatically. More generally, if $I$ is equimultiple, i.e. $\ell=$ ht $I$, then $I$ satisfies $G_{\ell}$. This condition is rather mild and there are many classes of ideals that would satisfy this condition (see for example CPU1, CPU2]).

(c) $K$ is a geometric $s$-residual intersection of an ideal $I$ if there exists an $s$-generated ideal $\mathfrak{a}$ with $K=\mathfrak{a}: I$ and ht $K \geq s \geq$ ht $I=g$ and ht $(I+K) \geq$ $s+1$. Residual intersections are a generalization of linkage theory when $R$ is a local Gorenstein ring or $I$ is an unmixed ideal. In this case if $s=g$, then for $K$ to be a $g$-residual intersection of $I$ is equivalent to $K$ being linked to $I$ since the ideal $\mathfrak{a}$ will be a complete intersection. This topic has been studied in great detail by various authors (see [CEU], [JU], [U]).

(d) $I$ is weakly $s$-residually $\mathrm{S}_{2}$ if $R / K$ satisfies Serre's condition $\mathrm{S}_{2}$ for every geometric $i$-residual intersection with $i \leq s$. Again we remark here that if $I$ is $\mathfrak{m}$-primary, or more generally equimultiple, then $I$ is weakly $(\ell-1)$-residually $\mathrm{S}_{2}$.

\section{Criteria AND formulas FOR THE $*$-CORE}

The following observation is an immediate consequence of the definition of the test ideal.

Observation 3.1. Let $R$ be a ring of characteristic $p>0$, with test ideal $\tau$. Let $I$ be an ideal. Then $\tau I \subseteq *$-core $(I)$. 
Proof. Let $J$ be an arbitrary *-reduction of $I$. Since $I \subseteq J^{*}$, it follows that $\tau \subseteq(J: I)$, and thus $\tau I \subseteq J$.

We will use the following characterization of minimal $*$-reductions from Vr2].

Theorem 3.2 ( $(\mathrm{Vr} 2$, Theorem 1.13]). Let $(R, \mathfrak{m})$ be an excellent normal local ring of characteristic $p>0$ and perfect residue field. Let $K \subseteq I$ be ideals, such that $K$ is generated by $n$ elements, where $n$ is the $*$-spread of $I$. Assume that I is tightly closed. The following are equivalent:

(a) $K$ is a minimal $*$-reduction of $I$.

(b) $I^{* s p} \cap K \subseteq \mathfrak{m} I$.

(c) $I=I^{* s p}+K$.

Using Theorem 3.2 we can describe explicitly all minimal $*$-reductions of an ideal.

Lemma 3.3. Let $(R, \mathfrak{m})$ be an excellent, normal local ring of characteristic $p>0$ and perfect residue field. Let $I$ be an ideal and let $J$ be a minimal $*$-reduction of I. Then we may write $I=J+\left(u_{1}, \ldots, u_{s}\right)$, with $u_{1}, \ldots, u_{s} \in J^{* s p}$. Suppose that $f_{1}, \ldots, f_{n}$ is a minimal system of generators of $J$ and let $K \subseteq I$. Then $K$ is a minimal $*$-reduction of $I$ if and only if $K=\left(f_{1}+v_{1}, f_{2}+v_{2}, \ldots, f_{n}+v_{n}\right)$, with $v_{1}, \ldots, v_{n} \in\left(u_{1}, \ldots, u_{s}\right)$.

Proof. First notice that $J$ is a minimal $*$-reduction of $I$ if and only if $J$ is a minimal *-reduction of $I^{*}$ and thus we may use Theorem 3.2 applied to $I^{*}$. Furthermore, any minimal generating set of $J$ can be extended to a minimal generating set for $I$ by [Ep Lemma 2.2]. Notice that $J \subseteq I \subseteq I^{*}=J+J^{* s p}$ by Theorem 3.2 and Ep, Lemma 3.4]. Hence we may write $I=J+\left(u_{1}, \ldots, u_{s}\right)$, where $u_{1}, \ldots, u_{s}$ may be chosen from $J^{* s p}$.

We check that for every $K=\left(f_{1}+v_{1}, \ldots, f_{n}+v_{n}\right)$ as in the statement, condition (b) in Theorem 3.2 holds. Indeed, if $c_{1}\left(f_{1}+v_{1}\right)+\ldots+c_{n}\left(f_{n}+v_{n}\right) \in I^{* s p}$, then we must have $c_{1} f_{1}+\ldots+c_{n} f_{n} \in I^{* s p} \cap J \subseteq \mathfrak{m} I$, where the last inclusion follows from [Vr2, Observation 1.11], noting that $J$ is a minimal *-reduction of $I$. Since $\sum_{i=1}^{n} c_{i} f_{i} \in \mathfrak{m} I$ and $\left\{f_{1}, \ldots, f_{n}\right\}$ is a minimal generating set for $J$, then $c_{i} \in \mathfrak{m}$ for all $i=1, \ldots, n$ and thus $I^{* s p} \cap K \subseteq \mathfrak{m} I$.

Conversely, let $K \subseteq I$ be a minimal *-reduction. Since $J$ is a minimal $*$-reduction of $I$, we know that the $*$-spread of $I$ is $n$, the minimal number of generators of $J$. Thus, $K$ must also be minimally generated by $n$ elements, and we can write $K=\left(g_{1}+v_{1}, \ldots, g_{n}+v_{n}\right)$, with $g_{i} \in\left(f_{1}, \ldots, f_{n}\right)$, and $v_{i} \in\left(u_{1}, \ldots, u_{s}\right)$ for all $i=1, \ldots, n$.

We claim that we must have $\left(g_{1}, \ldots, g_{n}\right)=\left(f_{1}, \ldots, f_{n}\right)$. Assuming this claim, we can write $\underline{f}=A \underline{g}$, with $A$ an invertible $n \times n$ matrix with entries in $R$, where $\underline{f}$ denotes the vector $\left(f_{1}, \ldots, f_{n}\right)^{\mathrm{T}}$. The entries of $A\left(g_{1}+v_{1}, \ldots, g_{n}+v_{n}\right)^{\mathrm{T}}$ are then generators for $K$, and they can be written in the form $f_{1}+v_{1}^{\prime}, \ldots, f_{n}+v_{n}^{\prime}$, where $\underline{v^{\prime}}=A \underline{v}$.

Assuming by contradiction that the claim is not true, we can choose a minimal system of generators $f_{1}^{\prime}, \ldots, f_{n}^{\prime}$ for $J$ such that $\left(g_{1}, \ldots, g_{n}\right) \subseteq \mathfrak{m} J+\left(f_{2}^{\prime}, \ldots, f_{n}^{\prime}\right)$. In order to achieve this, one can take $f_{1}^{\prime}, \ldots, f_{n}^{\prime}$ to be the pull-backs to $R$ of a vector space basis $\overline{f_{1}^{\prime}}, \ldots, \overline{f_{n}^{\prime}}$ of $J / \mathfrak{m} J$ obtained by extending a basis of the subspace spanned by $\overline{g_{1}}, \ldots, \overline{g_{n}}$. 
Since $K$ is a minimal $*$-reduction of $I$, we must have $f_{1}^{\prime} \in K^{*}$, and therefore $f_{1}^{\prime} \in\left(\mathfrak{m} f_{1}^{\prime}, f_{2}^{\prime}, \ldots, f_{n}^{\prime}, u_{1}, \ldots, u_{s}\right)^{*}$. It follows that $f_{1}^{\prime} \in\left(f_{2}^{\prime}, \ldots, f_{n}^{\prime}, u_{1}, \ldots, u_{s}\right)^{*}$ by the tight closure version of Nakayama's Lemma in [Ep, Proposition 2.1]. Since $u_{1}, \ldots, u_{s} \in J^{* s p}$, we see that in fact we must have $f_{1}^{\prime} \in\left(f_{2}^{\prime}, \ldots, f_{n}^{\prime}\right)^{*}$. Indeed, there exists $c \in R^{0}$ such that

$$
c f_{1}^{\prime q}=a_{2} f_{2}^{\prime q}+\ldots+a_{n} f_{n}^{\prime q}+b_{1} u_{1}^{q}+\ldots+b_{s} u_{s}^{q},
$$

for some $a_{i}, b_{j} \in R$ with $2 \leq i \leq n$ and $1 \leq j \leq s$. Since $u_{j} \in J^{* s p}$ for all $j$ there exists $q_{0}$ such that we can rewrite the above equation to obtain:

$$
\begin{aligned}
c^{2} f_{1}^{\prime q} & =c a_{2} f_{2}^{\prime q}+\ldots+c a_{n} f_{n}^{\prime q}+c b_{1} u_{1}^{q}+\ldots+c b_{s} u_{s}^{q} \\
& =a_{1}^{\prime} f_{1}^{\prime q}+\left(c a_{2}+a_{2}^{\prime}\right) f_{2}^{\prime q}+\ldots+\left(c a_{n}+a_{n}^{\prime}\right) f_{n}^{\prime q},
\end{aligned}
$$

with $a_{1}^{\prime}, \ldots, a_{n}^{\prime} \in \mathfrak{m}^{q / q_{0}}$. The conclusion follows by applying Proposition 2.2. This contradicts the fact that $f_{1}^{\prime}, \ldots, f_{n}^{\prime}$ are $*$-independent.

Theorem 3.4. Let $(R, \mathfrak{m})$ be a normal local ring of characteristic $p>0$ with perfect residue field. Let $I$ be an ideal and let $J$ be a minimal *-reduction of $I$. Then we may write $I=J+\left(u_{1}, \ldots, u_{s}\right)$, with $u_{1}, \ldots, u_{s} \in J^{* s p}$. Suppose that $f_{1}, \ldots, f_{n}$ is a minimal system of generators of $J$.

(a) If $\left(f_{1}, \ldots, \hat{f}_{i}, \ldots, f_{n}\right): f_{i} \subseteq(J: I)$ for all $i=1, \ldots, n$, and $u_{j}\left(J: u_{j}\right) \subseteq$ $(J: I) J$ for all $j=1, \ldots, s$, then $*$-core $(I) \subseteq(J: I) J$.

(b) If $u_{j}(J: I) \subseteq \mathfrak{m}(J: I) J$ for all $j=1, \ldots, s$, then $*$-core $(I) \supseteq(J: I) J$.

(c) If the assumptions of both part (a) and part (b) are satisfied, then

$$
\text { *-core }(I)=(J: I) J=(J: I) I .
$$

Proof. (a) Let $A_{1} f_{1}+\ldots+A_{n} f_{n} \in *$-core $(I)$. Fix $i \in\{1, \ldots, n\}$ and let $j \in$ $\{1, \ldots, s\}$. We wish to show that $A_{i} u_{j} \in J$. Since $j$ is arbitrary, it will follow that $A_{i} \in(J: I)$.

Let $J^{\prime}=\left(f_{1}, \ldots, f_{i-1}, f_{i}+u_{j}, f_{i+1}, \ldots, f_{n}\right)$ be another minimal $*$-reduction of $I$. We must have $A_{1} f_{1}+\ldots+A_{n} f_{n}=B_{1} f_{1}+\ldots+B_{i-1} f_{i-1}+B_{i}\left(f_{i}+u_{j}\right)+\ldots+B_{n} f_{n}$ for some $B_{1}, \ldots, B_{n} \in R$. Note we must have $B_{i} u_{j} \in u_{j}\left(J: u_{j}\right) \subseteq(J: I) J$ by assumption, and thus we can write $B_{i} u_{j}=C_{1} f_{1}+\ldots+C_{n} f_{n}$ with $C_{1}, \ldots, C_{n} \in$ $(J: I)$. Therefore $A_{i}-B_{i}-C_{i} \in\left(f_{1}, \ldots, \hat{f}_{i}, \ldots, f_{n}\right): f_{i} \subseteq(J: I)$ and, since $C_{i} \in$ $(J: I)$, then $A_{i}-B_{i} \in(J: I)$. Multiplying by $u_{j}$ we obtain $A_{i} u_{j}-B_{i} u_{j} \in(J: I) I$ and thus $A_{i} u_{j} \in(J: I) I \subseteq J$.

(b) Let $A_{1} f_{1}+\ldots+\bar{A}_{n} f_{n} \in(J: I) J$, with $A_{1}, \ldots, A_{n} \in(J: I)$, and let $J^{\prime}$ be another minimal $*$-reduction of $I$. Then by Lemma 3.3 we can assume that $J^{\prime}=\left(f_{1}+v_{1}, \ldots, f_{n}+v_{n}\right)$, where $v_{1}, \ldots, v_{n} \in\left(u_{1}, \ldots, u_{s}\right)$. We wish to find $B_{1}, \ldots, B_{n} \in(J: I)$ such that

$$
A_{1} f_{1}+\ldots+A_{n} f_{n}=B_{1}\left(f_{1}+v_{1}\right)+\ldots+B_{n}\left(f_{n}+v_{n}\right) .
$$

Write $(J: I)=\left(g_{1}, \ldots, g_{l}\right)$, and $A_{i}=\sum_{j=1}^{l} \alpha_{i j} g_{j}, B_{i}=\sum_{j=1}^{l} y_{i j} g_{j}$, where $y_{i j}$ are unknowns. By assumption, we can write $v_{i} g_{k}=\sum_{\nu=1}^{n} \sum_{j=1}^{l} \gamma_{i k j \nu} g_{j} f_{\nu}$, with $\gamma_{i k j \nu} \in \mathfrak{m}$ for all $i=1, \ldots, n$ and all $k=1, \ldots, l$.

A sufficient condition for equation (1) to hold is that the coefficients of $f_{\nu}$ on each side of the equation are the same for all $\nu=1, \ldots, n$, which leads to the system 
of linear equations

$$
\sum_{j=1}^{l} y_{\nu j} g_{j}+\sum_{j=1}^{l} \sum_{i=1}^{n} \sum_{k=1}^{l} y_{i k} \gamma_{i k j \nu} g_{j}=\sum_{j=1}^{l} \alpha_{\nu j} g_{j} \quad \text { for all } \nu=1, \ldots, n .
$$

A further sufficient condition is obtained by identifying the coefficients of each $g_{j}$ on the two sides of the equations (2). The following system of linear equations is thus obtained:

$$
(\nu, j): \quad y_{\nu j}+\sum_{i=1}^{n} \sum_{k=1}^{l} y_{i k} \gamma_{i k j \nu}=\alpha_{\nu j} \quad \text { for all } \nu=1, \ldots, n, j=1, \ldots, l .
$$

This is a system of $n l$ equations with $n l$ unknowns. Note that each $y_{\nu j}$ appears with a unit coefficient in equation $(\nu, j)$ and with a coefficient in $\mathfrak{m}$ in all the other equations, and therefore the matrix of coefficients can be row-reduced to the identity matrix, showing that there exists a unique solution for the system.

Corollary 3.5. With the same setup as in Theorem 3.4 if the assumptions of both part $(a)$ and part $(b)$ are satisfied, *-core $(I)$ is obtained as a finite intersection of minimal *-reductions, namely

$$
* \text {-core }(I)=J \cap\left(\bigcap_{i, j} J_{i, j}\right),
$$

where $J=\left(f_{1}, \ldots, f_{n}\right)$ and $J_{i, j}:=\left(f_{1}, \ldots, f_{i-1}, f_{i}+u_{j}, f_{i+1}, \ldots f_{n}\right)$, for all $i \in$ $\{1, \ldots, n\}$ and $j \in\{1, \ldots, s\}$.

Proof. The proof of part (a) of Theorem 3.4 shows that $J \cap\left(\bigcap_{i, j} J_{i, j}\right) \subseteq(J: I) J$, while part (b) of Theorem 3.4 shows that $(J: I) J \subseteq *$-core $(I)$. The conclusion follows, since $*$-core $(I) \subseteq J \cap\left(\bigcap_{i, j} J_{i, j}\right)$ by the definition of the $*$-core.

Remark 3.6. Let $(R, \mathfrak{m})$ be a Cohen-Macaulay, excellent, normal local ring with $\operatorname{dim} R \geq 2$, characteristic $p>0$ and perfect residue field. Let $\tau$ be the test ideal of $R$. The normality assumption implies that $\operatorname{depth} \tau \geq 2$. This is because the defining ideal of the non-regular locus has height at least two, and [HH2, Theorem 6.2] shows that we can pick a regular sequence consisting of two elements $c, d$ in this ideal and replace them by sufficiently large powers in order to obtain a regular sequence of length two consisting of test elements. Thus we may assume that one can choose a system of parameters $x_{1}, \ldots, x_{d}$ for $R$ with $x_{1}, x_{2} \in \tau$.

Theorem 3.7. Let $(R, \mathfrak{m})$ be a Cohen-Macaulay, excellent, normal local ring with $\operatorname{dim} R \geq 2$, characteristic $p>0$, and perfect residue field. Let $x_{1}, \ldots, x_{d}$ be part of a system of parameters, with $x_{1}, x_{2} \in \tau$, where $\tau$ is the test ideal of $R$. Let $I$ be an ideal and suppose $J=\left(x_{1}^{t}, x_{2}^{t}, x_{3}, \ldots, x_{d}\right) \subseteq I \subseteq J^{*}$, where $t$ is a positive integer. If $t \geq 2$, then $*$-core $(I) \subseteq(J: I) J=(J: I) I$; and when $t \geq 3$, then equality is obtained.

Proof. Let $I=J+\left(u_{1}, \ldots, u_{s}\right)$, where $u_{i} \in I^{* s p}$ for all $1 \leq i \leq s$. We need to see that all the hypotheses listed in part (a) and part (b) of Theorem 3.4 hold.

To confirm that the hypotheses of part (a) hold, notice that

$$
\left(x_{1}^{t}, x_{2}^{t}, x_{3}, \ldots, \widehat{x_{i}}, \ldots, x_{d}\right): x_{i}=\left(x_{1}^{t}, x_{2}^{t}, x_{3}, \ldots, \widehat{x_{i}}, \ldots, x_{d}\right) \subseteq J \subseteq(J: I) .
$$


For the second hypothesis in part (a), note that

$$
J^{*} \subseteq J: \tau \subseteq J:\left(x_{1}, x_{2}\right)=\left(x_{1}^{t}, x_{2}^{t}, x_{3}, \ldots, x_{d},\left(x_{1} x_{2}\right)^{t-1}\right) .
$$

We claim that $J^{* s p} \subseteq \mathfrak{m} J+\left(x_{1} x_{2}\right)^{t-1}$. To verify this, suppose that $f \in J^{* s p}$. Since $J^{*}=J+J^{* s p} \subseteq J+\left(x_{1} x_{2}\right)^{t-1}$, we may write $f=g+u\left(x_{1} x_{2}\right)^{t-1}$ for some $g \in J$ and $u \in R$. Since $f, g \in J^{*}$, then $u\left(x_{1} x_{2}\right)^{t-1} \in J^{*}$. Then there exists $c \in R^{0}$ such that for all large powers $q$ of $p$ we have $c u^{q}\left(x_{1} x_{2}\right)^{q(t-1)} \in J^{[q]}=$ $\left(x_{1}^{q t}, x_{2}^{q t}, x_{3}^{q}, \ldots, x_{d}^{q}\right)$. Therefore

$$
c u^{q} \in\left(x_{1}^{q t}, x_{2}^{q t}, x_{3}^{q}, \ldots, x_{d}^{q}\right):\left(x_{1} x_{2}\right)^{q(t-1)}=\left(x_{1}^{q}, x_{2}^{q}, x_{3}^{q}, \ldots, x_{d}^{q}\right) .
$$

Hence

$$
\begin{aligned}
c u^{q}\left(x_{1} x_{2}\right)^{q(t-1)} & \in\left(x_{1} x_{2}\right)^{q(t-1)}\left(x_{1}^{q}, x_{2}^{q}, x_{3}^{q}, \ldots, x_{d}^{q}\right) \\
& =\left(x_{1}^{q t} x_{2}^{q(t-1)}, x_{2}^{q t} x_{1}^{q(t-1)},\left(x_{1} x_{2}\right)^{q(t-1)} x_{3}^{q}, \ldots,\left(x_{1} x_{2}\right)^{q(t-1)} x_{d}^{q}\right) \\
& \subseteq\left(x_{1}^{q}, x_{2}^{q}\right)\left(x_{1}^{q t}, x_{2}^{q t}, x_{3}^{q}, \ldots, x_{d}^{q}\right)=\left(x_{1}^{q}, x_{2}^{q}\right) J^{[q]} \subseteq \mathfrak{m}^{[q]} J^{[q]},
\end{aligned}
$$

where the penultimate inclusion holds as long as $t \geq 2$. Therefore $u\left(x_{1} x_{2}\right)^{(t-1)} \in$ $(\mathfrak{m} J)^{*}$, and by definition of the special tight closure we have $u\left(x_{1} x_{2}\right)^{(t-1)} \in J^{* s p}$. As $f \in J^{* s p}$, then $g \in J^{* s p}$ as well. But $g \in J$ also and thus $g \in J \cap J^{* s p}=\mathfrak{m} J$, by Vr1, Proposition 4.2]. Therefore $J^{* s p} \subseteq \mathfrak{m} J+\left(x_{1} x_{2}\right)^{t-1}$. Since the hypotheses in Theorem 3.4 are unaffected when the $u_{i}$ 's are replaced by $u_{i}+w_{i}$ with $w_{i} \in \mathfrak{m} J$, we may assume without loss of generality that we can write $u_{j}=\left(x_{1} x_{2}\right)^{t-1} u_{j}^{\prime}$.

We have

$$
u_{j}\left(J: u_{j}\right)=\left(x_{1} x_{2}\right)^{t-1} u_{j}^{\prime}\left(\left(x_{1}, x_{2}, x_{3}, \ldots, x_{d}\right): u_{j}^{\prime}\right) \subseteq\left(x_{1}, x_{2}\right) J \subseteq(J: I) J .
$$

The last inclusion follows from the fact that $\left(x_{1}, x_{2}\right) \subseteq \tau \subseteq(J: I)$.

Finally we establish that the hypothesis in part (b) holds. As above, we can write $u_{j}=\left(x_{1} x_{2}\right)^{t-1} u_{j}^{\prime}$, and thus $(J: I) \subseteq\left(x_{1}, x_{2}, x_{3}, \ldots, x_{d}\right):\left(u_{1}^{\prime}, \ldots, u_{s}^{\prime}\right)$. Therefore

$$
u_{j}(J: I) \subseteq\left(x_{1} x_{2}\right)^{t-1}\left(x_{1}, x_{2}, x_{3}, \ldots, x_{d}\right) \subseteq\left(x_{1}^{t-1}, x_{2}^{t-1}\right) J \subseteq \mathfrak{m} J(J: I),
$$

where the last inclusion follows from the fact that $\left(x_{1}^{t-1}, x_{2}^{t-1}\right) \subseteq \mathfrak{m}\left(x_{1}, x_{2}\right) \subseteq \mathfrak{m}(J: I)$ when we take $t \geq 3$.

The following observation can be obtained as a corollary of Theorem 3.7 and FV, Theorem 4.8], using [CPU2, Theorem 2.6]. Nonetheless, we give an elementary direct proof.

Observation 3.8. Let $R$ be a Cohen-Macaulay, excellent, normal local ring with $\operatorname{dim} R \geq 2$, characteristic $p>0$, and perfect residue field. Let $x_{1}, \ldots, x_{d}$ be part of a system of parameters with $x_{1}, x_{2} \in \tau$, where $\tau$ is the test ideal of $R$. Let $I$ be an ideal and suppose $J=\left(x_{1}^{t}, x_{2}^{t}, x_{3}, \ldots, x_{d}\right) \subseteq I \subseteq J^{*}$, where $t \geq 2$. Then $r_{J}(I)=1$.

Proof. As in the proof of Theorem 3.7 we can write $I=J+\left(x_{1} x_{2}\right)^{t-1}\left(u_{1}^{\prime}, \ldots, u_{s}^{\prime}\right)$. Thus it suffices to see that $\left(x_{1} x_{2}\right)^{t-1} u_{i}^{\prime}\left(x_{1} x_{2}\right)^{(t-1)} u_{j}^{\prime} \in J^{2}$ for all $i, j \in\{1, \ldots, s\}$. This is immediate, since $\left(x_{1} x_{2}\right)^{2 t-2} \in\left(x_{1}^{t} x_{2}^{t}\right) \subseteq J^{2}$ as long as $t \geq 2$.

Corollary 3.9. Let $(R, \mathfrak{m})$ be a Cohen-Macaulay, excellent, normal local ring with $\operatorname{dim} R \geq 2$, characteristic $p>0$ and perfect residue field. Let $x_{1}, \ldots, x_{d}$ be a system of parameters with $x_{1}, x_{2} \in \tau$, where $\tau$ is the test ideal of $R$. Let $I$ be an ideal and suppose $\left(x_{1}^{t}, x_{2}^{t}, x_{3}, \ldots, x_{d}\right) \subseteq I \subseteq\left(x_{1}^{t}, x_{2}^{t}, x_{3}, \ldots, x_{d}\right)^{*}$, where $t \geq 2$. Then $\operatorname{gr}_{I} R$ is Cohen-Macaulay. 
Proof. Notice that with the above conditions $I$ is an $\mathfrak{m}$-primary ideal and satisfies $G_{\ell}$, where $\ell$ is the analytic spread. Also, the condition depth $R / I^{j} \geq d-g-j+1$ for all $1 \leq j \leq \ell-g+1$, where $g=\operatorname{ht}(I)$, is immediately satisfied and by Observation 3.8 $r(I)=1$. Therefore, by [JU, Theorem 3.1] we have $\operatorname{gr}_{I}(R)$ is Cohen-Macaulay.

Theorem 3.10. Let $(R, \mathfrak{m})$ be a normal local ring of characteristic $p>0$ and perfect residue field. Let $I$ be an ideal and suppose that the test ideal is $\mathfrak{m}$. Then *-core $(I)=(J: I) J=(J: I) I$ for any minimal $*$-reduction $J$ of $I$.

Proof. Since $\tau \subseteq(J: I)$, we have $(J: I)=\mathfrak{m}$ or $(J: I)=R$. Note that $(J: I)=R$ is equivalent to $I=J$, which means that $I$ is the only minimal $*$-reduction of $I$, and thus the conclusion holds in this case.

Hence, we may assume that $(J: I)=\mathfrak{m}$. We have $(J: I) J \subseteq *$-core $(I)$ by Observation 3.1. In order to verify the other inclusion, we will check that the assumptions in part (a) of Theorem 3.4 hold. The fact that

$$
\left(f_{1}, \ldots, \hat{f}_{i}, \ldots, f_{n}\right): f_{i} \subseteq \mathfrak{m}
$$

is immediate, since $f_{1}, \ldots, f_{n}$ is a minimal system of generators of $J$. It remains to check that $u(J: I) \subseteq \mathfrak{m} J$, for $u \in J^{* s p}$.

Since $u \in J^{* s p} \cap I$, then $u(J: I) \subseteq J^{* s p}$. On the other hand, $u(J: I) \subseteq J$ and thus $u(J: I) \subseteq J^{* s p} \cap J=\mathfrak{m} J$, where the last equality holds by [Vr1, Proposition 4.2].

Lemma 3.11. Let $(R, \mathfrak{m})$ be an excellent, analytically irreducible local ring of characteristic $p>0$ and $\mathfrak{m}$-primary test ideal $\tau$. Let $f_{1}, \ldots, f_{n}$ be $*$-independent elements. Let $J=\left(f_{1}, \ldots, f_{n}\right)$ and consider $I=\left(f_{1}, \ldots, f_{n}, u_{1}, \ldots, u_{s}\right)$, where $u_{1}, \ldots, u_{s} \in J^{* s p}$. Then there exists $q_{0}$ sufficiently large such that for all $q \geq q_{0}$ the following hold:

(a) $\left(f_{1}^{q}, \ldots, \hat{f}_{i}^{q}, \ldots, f_{n}^{q}\right): f_{i}^{q} \subseteq \tau$ for all $i=1, \ldots, n$, and

(b) $u_{k}^{q}\left(J^{[q]}: u_{k}^{q}\right) \subseteq \mathfrak{m} \tau J^{[q]}$ for all $k=1, \ldots, s$.

Proof. Part (a) follows from Proposition 2.2,

It remains to prove part (b). Fix $j$ sufficiently large so that $\left(\tau^{j}: \tau\right) \subseteq \mathfrak{m} \tau$. This is possible by the Artin-Rees Lemma, since $\left(\tau^{j}: \tau\right) \subseteq \tau^{j}: d$ for a fixed nonzerodivisor $d \in \tau$, and $F d \in \tau^{j}$ implies that $F d \in(d) \cap \tau^{j} \subseteq d \tau^{j-h}$, i.e. $F \in \tau^{j-h}$, where $h$ is a constant. Since $\tau$ is $\mathfrak{m}$-primary, we can use Proposition 2.2 to find a $q_{0}$ so that $\left(f_{1}^{q}, \ldots, \hat{f}_{i}^{q}, \ldots, f_{n}^{q}\right): f_{i}^{q} \subseteq \tau^{j}$ for all $q \geq q_{0}$ and for all $i=1, \ldots, n$.

Since $u_{k} \in J^{* s p}$, we can choose $q \gg 0$ so that $u_{k}^{q} \in\left(\tau^{j} J^{[q]}\right)^{*} \subseteq\left(\tau^{j} J^{[q]}\right): \tau$. Let $g \in\left(J^{[q]}: u_{k}^{q}\right)$ and write $g u_{k}^{q}=c_{1} f_{1}^{q}+\ldots+c_{n} f_{n}^{q}$. Multiplying by $t \in \tau$ yields $t g u_{k}^{q}=\left(t c_{1}\right) f_{1}^{q}+\ldots+\left(t c_{n}\right) f_{n}^{q}$. But on the other hand we have $t u_{k}^{q}=d_{1} f_{1}^{q}+\ldots+d_{n} f_{n}^{q}$, with $d_{1}, \ldots, d_{n} \in \tau^{j}$. It follows that $t c_{i}-g d_{i} \in\left(f_{1}^{q}, \ldots, \hat{f}_{i}^{q}, \ldots, f_{n}^{q}\right): f_{i}^{q} \subseteq \tau^{j}$, and therefore $c_{i} \in\left(\tau^{j}: \tau\right) \subseteq \mathfrak{m} \tau$.

Theorem 3.12. Let $(R, \mathfrak{m})$ be a normal local ring of characteristic $p>0$, with perfect residue field and $\mathfrak{m}$-primary test ideal $\tau$. Let $I$ be an ideal and let $J \subseteq I$ be a minimal $*$-reduction of $I$. Then $*$-core $\left(I^{[q]}\right)=\left(J^{[q]}: I^{[q]}\right) J^{[q]}$ for all $q=p^{e} \gg 0$. 
Proof. Let $I=J+\left(u_{1}, \ldots, u_{s}\right)$, where $u_{j} \in J^{* s p}$ for all $j=1, \ldots, s$. We need to prove $*$-core $\left(I^{[q]}\right)=\left(J^{[q]}: I^{[q]}\right) J^{[q]}$ by showing the hypotheses of Theorem 3.4 hold when $I, J$ are replaced by $I^{[q]}, J^{[q]}$ respectively.

First note that by Lemma 3.11 part (a), $\left(f_{1}^{q}, \ldots, \hat{f}_{i}^{q}, \ldots, f_{n}^{q}\right): f_{i}^{q} \subseteq \tau \subseteq$ $\left(J^{[q]}: I^{[q]}\right)$ showing that the first hypothesis of part (a) in Theorem 3.4 is satisfied. Using Lemma 3.11 part (b) we have that $u_{j}^{q}\left(J^{[q]}: u_{j}^{q}\right) \subseteq \mathfrak{m} \tau J^{[q]} \subseteq\left(J^{[q]}: I^{[q]}\right) J^{[q]}$ for all $j=1, \ldots, s$, which shows that the hypotheses of Theorem 3.4 part(a) hold.

In order to verify that the hypothesis of Theorem 3.4part (b) holds we note that $\tau \subseteq\left(J^{[q]}: I^{[q]}\right)$. Thus the result follows by Lemma 3.11 part (b).

\section{EXAMPLES}

The first example was an inspiration for Theorem 3.7 although the ring is not normal.

Example 4.1. Let $R=k[[x, y, z]] /(x y z)$, where $k$ is a field of characteristic $p>0$ and $I=(x, y z)$. By $\left[\mathrm{Va}\right.$, Theorem 3.7], $\tau=(x y, x z, y z)$ and $I=(x+a y z)^{*}$ for any $0 \neq a \in k$. Let $J=(x+a y z)$, for some $0 \neq a \in k$. Note that

$$
((x+a y z): I) J=\left(x^{2}, y^{2} z^{2}\right)=\bigcap_{a \in k}(x+a y z)=* \operatorname{core}(I) .
$$

If the parameter ideal is not sufficiently embedded for Theorem 3.7 to apply, the following example shows that $*$-core $(I) \neq(J: I) J$.

Example 4.2. Let $R=k[x, y, z] /\left(x^{5}+y^{5}+z^{5}\right)$, where $k$ is a perfect field of characteristic $p>0$ such that $p \neq 5$. Let $J=(y, z)$ and set $u=x^{2}$ and $I=J+(u)$. Note that $u \in J^{*}$ as can be seen from the fact that $\tau=\mathfrak{m}^{3}$ and $u \in\left(J: \mathfrak{m}^{3}\right)$. The assumptions in both part (a) and part (b) of Theorem 3.4 fail, and $(J: I) J \nsubseteq J^{\prime}=\left(y+x^{2}, z\right)$, which is a minimal $*$-reduction of $I$.

We are going to use the following result of Brenner to give another example where the equality $*$-core $(I)=(J: I) J$ is not always obtained when the assumptions of Theorem 3.4 do not hold.

Theorem 4.3 ([Br. Example 5.2]). If $k$ is an algebraically closed field,

$$
R=\frac{k[x, y, z]}{\left(x^{d}+y^{d}+z^{d}\right)}
$$

where $d$ is not divisible by the characteristic of $k$, and $f=x^{d_{1}}, g=y^{d_{2}}, h=z^{d_{3}}$ are such that $d_{i} \leq d$ for $i \in\{1,2,3\}$, and $d_{1}+d_{2}+d_{3} \leq 2 d$, then $R_{\geq d} \subseteq(f, g, h)^{*}$.

Note that if $R$ is a graded ring, $J$ is a homogeneous ideal, and $u \in J^{*}$ has degree larger than the degrees of the generators of $J$, then $u \in J^{* s p}$.

Example 4.4. Let $R=k[x, y, z] /\left(x^{10}+y^{10}+z^{10}\right)$, where $k$ is an algebraically closed field of characteristic $p>0$ such that $p \neq 2,5$. Let $J=\left(x^{5}, y^{7}, z^{8}\right)$ and $u=x y^{3} z^{6}$. By Theorem 4.3. $u$ is in the tight closure of $J$. Define $I=J+(u)$, and note 
that $J$ is a minimal $*$-reduction of $I$. The assumption that $u(J: I) \subseteq(J: I) J$ fails in Theorem 3.4 because $(J: I)=\left(x^{4}, y^{4}, z^{2}\right)$ and $z^{2} u \notin(J: I) J$. We have that $(J: I) J \nsubseteq *$-core $(I)$. More specifically, $x^{5} z^{2} \in(J: I) J$, but it is not in $J^{\prime}=\left(x^{5}+x u, y^{7}+y u, z^{8}+z u\right)$, which is a minimal $*$-reduction of $I$.

\section{REFERENCES}

[Ab] Aberbach, I., Extensions of weakly and strongly F-rational rings by flat maps, J. Algebra, 241 (2001), 799-807. MR.1843326 (2002f:13008)

[Br] Brenner, H., Computing the tight closure in dimension two, Math. Comp., 74 (2005), 1495-1518. MR2137014 (2006h:13008)

[CEU] Chardin, M., Eisenbud, D., Ulrich, B., Hilbert functions, residual intersections, and residually $\mathrm{S}_{2}$ ideals, Compositio Math., 125 (2001), 193-219. MR1815393(2002g:13034)

[CPU1] Corso, A., Polini, C., Ulrich, B., The structure of the core of ideals, Math. Ann., 321 (2001), 89-105. MR1857370 (2002j:13005)

[CPU2] Corso, A., Polini, C., Ulrich, B., Core and residual intersections of ideals, Trans. Amer. Math. Soc., 354 (2002), 2579-2594. MR1895194 (2003b:13035)

[Ep] Epstein, N., A tight closure analogue of analytic spread, Math. Proc. Camb. Phil. Soc., 139 (2005), 371-383. MR2168094(2006e:13003)

[EV] Epstein, N., Vraciu, A., A length characterization of *-spread, Osaka J. Math., 45 (2008), 445-456. MR 2441949 (2009d:13003)

[FV] Fouli, L., Vassilev, J., The cl-core of an ideal, Math. Proc. Camb. Phil. Soc., 149 (2010), 247-262. MR2670215

[HH1] Hochster, M., Huneke, C., Tight closure, invariant theory, and the Briançon-Skoda theorem, J. Amer. Math. Soc., 3 (1990), 31-116. MR.1017784 (91g:13010)

[HH2] Hochster, M., Huneke, C., F-regularity, test elements, and smooth base change, Trans. Amer. Math. Soc., 346 (1994), 1-62. MR.1273534 (95d:13007)

[Hu] Huneke, C., Tight Closure and Its Applications, CBMS Regional Conference Series in Mathematics, 88, American Math. Soc., Providence, RI, 1996. MR1377268|(96m:13001)

[HS1] Huneke, C., Swanson, I., Cores of ideals in 2-dimensional regular local rings, Michigan Math. J., 42 (1995), 193-208. MR.1322199 (96j:13021)

[HT] Huneke, C., Trung, N., On the core of ideals, Compos. Math., 141 (2005), 1-18. MR 2099767 (2005g:13003)

[HV] Huneke, C., Vraciu, A., Special tight closure, Nagoya Math. J., 170 (2003), 175-183. MR.1994893 (2004e:13008)

[HySm1] Hyry, E., Smith, K., On a non-vanishing conjecture of Kawamata and the core of an ideal, Amer. J. Math., 125 (2003), 1349-1410. MR2018664 (2006c:13036)

[HySm2] Hyry, E., Smith, K., Core versus graded core, and global sections of line bundles, Trans. Amer. Math. Soc., 356 (2004), 3143-3166. MR2052944 (2005g:13007)

[JU] Johnson, M., Ulrich, B., Artin-Nagata properties and Cohen-Macaulay associated graded rings, Compositio Math., 103 (1996), 7-29. MR.1404996 (97f:13006)

[NR] Northcott, D. G. and Rees D., Reductions of ideals in local rings, Proc. Camb. Phil. Soc., 50 (1954), 145-158. MR0059889 (15:596a)

[PU] Polini, C., Ulrich, B., A formula for the core of an ideal, Math. Ann., 331 (2005), 487-503. MR 2122537 (2006k:13020)

[RS] Rees, D., Sally, J., General elements and joint reductions, Michigan Math. J., 35 (1988), 241-254. MR 959271 (89h:13034)

[U] Ulrich, B., Artin-Nagata properties and reductions of ideals, Contemp. Math., 159, American Math. Soc., Providence, RI, 1994, 373-400. MR1266194(95a:13017)

[Va] Vassilev, J., Test ideals in quotients of F-finite regular local rings, Trans. Amer. Math. Soc., 350 (1998), 4041-4051. MR.1458336 (98m:13009)

[Vr1] Vraciu, A., *-independence and special tight closure, J. Algebra, 249 (2002), 544-565. MR:1901172 (2003d:13003)

[Vr2] Vraciu, A., Chains and families of tightly closed ideals, Bull. London Math. Soc., 38 (2006), 201-208. MR 2214472 (2007e:13009) 
Department of Mathematical Sciences, New Mexico State University, Las Cruces, New Mexico 88003

E-mail address: lfouli@math.nmsu.edu

Department of Mathematics and Statistics, University of New Mexico, Albuquerque, New MeXico 87131

E-mail address: jvassil@math.unm.edu

Department of Mathematics, University of South Carolina, Columbia, South CaroLINA 29208

E-mail address: vraciu@math.sc.edu 